\title{
Leading coefficients of Kazhdan-Lusztig polynomials for Deodhar elements
}

\author{
Brant C. Jones
}

Received: 8 November 2007 / Accepted: 21 February 2008 / Published online: 6 March 2008

(C) Springer Science+Business Media, LLC 2008

\begin{abstract}
We show that the leading coefficient of the Kazhdan-Lusztig polynomial $P_{x, w}(q)$ known as $\mu(x, w)$ is always either 0 or 1 when $w$ is a Deodhar element of a finite Weyl group. The Deodhar elements have previously been characterized using pattern avoidance in Billey and Warrington (J. Algebraic Combin. 13(2):111136, 2001) and Billey and Jones (Ann. Comb. 2008, to appear). In type $A$, these elements are precisely the 321-hexagon avoiding permutations. Using Deodhar's algorithm (Deodhar in Geom. Dedicata 63(1):95-119, 1990), we provide some combinatorial criteria to determine when $\mu(x, w)=1$ for such permutations $w$.
\end{abstract}

Keywords Kazhdan-Lusztig polynomial $\cdot 321$-hexagon $\cdot 0$-1 conjecture $\cdot$ Pattern avoidance

\section{Introduction to the $0-1$ property}

In this paper we show that when $w$ is a Deodhar element of a finite Weyl group, the coefficient of $q^{\frac{1}{2}(l(w)-l(x)-1)}$ in the Kazhdan-Lusztig polynomial $P_{x, w}(q)$ is always either 0 or 1 . This notorious coefficient is known as $\mu(x, w)$ in the literature and corresponds to the term of highest possible degree in $P_{x, w}(q)$. The $\mu(x, w)$ values appear in multiplication formulas for the Kazhdan-Lusztig basis elements $\left\{C^{\prime}{ }_{w}\right\}$ of the Hecke algebra and are used in the construction of Kazhdan-Lusztig graphs and cells which in turn are used to construct Hecke algebra representations. This was first done in [4] and is described in detail for type $A$ by [5]. Note that the $\mu$ coefficients control the recursive structure of the Kazhdan-Lusztig polynomials and computing

The author received support from NSF grants DMS-9983797 and DMS-0636297.

B.C. Jones $(\varangle)$

Department of Mathematics, University of California, One Shields Avenue, Davis, CA 95616, USA

e-mail: brant@math.ucdavis.edu

url: http://www.math.ucdavis.edu/ brant/ 
$\mu(x, w)$ is not known to be any easier than computing the entire Kazhdan-Lusztig polynomial $P_{x, w}(q)$. The Deodhar elements have been characterized previously by pattern avoidance as those that are [321]-hexagon avoiding permutations in [1] and for the other finite Weyl groups in [2].

Our main result is motivated by the following open problem.

Question 1.1 Fix a finite Coxeter group $W$. Is there a simple characterization of the elements $w \in W$ such that $\mu(x, w) \in\{0,1\}$ for all $x \in W$ ?

When all of the elements $w$ in a left cell $\mathcal{C}$ of the finite Weyl group $W$ satisfy $\mu(x, w) \in\{0,1\}$ for all $x \in W$, then Kazhdan-Lusztig's construction of the representation of $W$ associated to $\mathcal{C}$ depends only on the underlying graph structure of the $W$-graph, rather than the edge-labeling of the graph by $\mu$ coefficients. See [4] or Chapter 6 of [5] for details.

Until fairly recently, it was conjectured that all $\mu(x, w)$ for type $A$ were either 0 or 1. See [6] for some history about this conjecture and an example in $S_{10}$ that shows the conjecture to be false. On the other hand, if $w$ and $x$ are elements of a finite Weyl group then under each of the hypotheses below we have $\mu(x, w) \in\{0,1\}$.

(1) Suppose $w \in S_{n}$, the symmetric group with $n \leq 9$.

(2) Suppose there exists a simple reflection $s$ with $s w<w$ and $s x>x$ in Bruhat order.

(3) Suppose $w$ is a covexillary permutation (equivalently, the 1-line notation for $w$ avoids the permutation pattern [3412]).

(4) Suppose $w$ corresponds to a smooth Schubert variety.

(5) Suppose $w$ and $x$ are fully commutative elements of types $A, D$, or $E$.

(6) Suppose $w$ and $x$ are Boolean elements of types $A, B$, or $F$ defined in [15].

(7) Suppose $w, x \in S_{n}$, the symmetric group, with $a(x)<a(w)$ where $a: S_{n} \rightarrow \mathbb{N}$ is the function defined by Lusztig in [7].

We have (1) from a difficult verification due to [6]. The standard recursive formula for $P_{x, w}$ from [4] together with (2) implies that $\mu(x, w)=0$ or else $l(w)-l(x)=1$ in which case $\mu(x, w) \in\{0,1\}$. This holds for all Coxeter groups. We have (3) from a formula developed in [8] and [9] for $P_{x, w}$ using certain edge labeled trees. When the Schubert variety associated to $w$ is smooth, we have by [10] that $P_{x, w}(q)=1$ for all $x$, proving (4). In type $A$, we mention that $w$ is $\{[3412],[4231]\}$-avoiding if and only if $w$ is smooth by [11-13]. In [14], (5) was observed for types $A, D$ and $E$. Result (6) follows from a closed product formula of [15, Theorem 5.4] and holds in other Coxeter types as well. The main result of [16] is (7). Note that (5), (6) and (7) require a restriction on both $x$ and $w$.

Our main result in Theorem 1.2 adds a new set of elements to the list above and will follow from Theorems 4.1, 5.4 and 6.1 as well as Corollary 4.3 below.

Theorem 1.2 If $w$ is a Deodhar element of a finite Weyl group $W$ and $x \in W$ then $\mu(x, w) \in\{0,1\}$.

In Section 2 we describe the main tools used in the proof of Theorem 1.2. We will begin by proving Theorem 1.2 for type $A$ in Sections 3 and 4. This proof also holds 
for type $B$ since the unlabeled Coxeter graph of type $B$ is the same as the unlabeled Coxeter graph of type $A$. In Section 5 we describe how to reduce to type $A$ from type $D$. Finally, we verify the theorem for the exceptional finite Weyl groups by computer in Section 6.

Although the first $\mu$ coefficient that is larger than 1 does not appear in type $A$ until rank 9 , there are $\mu$ coefficients as large as 4 in $D_{6}$ and as large as 6 in $E_{6}$. This can be verified using the program Coxeter 1.01 of du Cloux [17].

\section{Background and introduction to $\mu$-masks}

We assume that the reader is familiar with the basic definitions and results from Coxeter groups and Kazhdan-Lusztig theory discussed in [18] or [5], as well as the standard notions of pattern avoidance reviewed in [2]. Our main tools in this paper are Deodhar's combinatorial setting [3] for Kazhdan-Lusztig polynomials and the decorated heaps introduced in [1] and [2] to encode the masks of Deodhar's framework. We refer the reader to the introductions of [1] and [2] for a more leisurely discussion of these topics.

\subsection{Deodhar's Theorem}

Let $w$ be an element of a Coxeter group $W$ and fix a reduced expression $\mathrm{w}=$ $\mathrm{w}_{1} \mathrm{w}_{2} \cdots \mathrm{w}_{k}$ for $w$, so each $\mathrm{w}_{i}$ is a Coxeter generator. Define a mask $\sigma$ associated to the reduced expression $\mathrm{w}$ to be any binary vector $\left(\sigma_{1}, \ldots, \sigma_{k}\right)$ of length $k=l(w)$. Every mask corresponds to a subexpression of $\mathbf{w}$ defined by $\mathbf{w}^{\sigma}=\mathrm{w}_{1}^{\sigma_{1}} \cdots \mathrm{w}_{k}^{\sigma_{k}}$ where

$$
\mathrm{w}_{j}^{\sigma_{j}}= \begin{cases}\mathrm{w}_{j}, & \text { if } \sigma_{j}=1, \\ \mathrm{id}, & \text { if } \sigma_{j}=0\end{cases}
$$

Each $\mathbf{w}^{\sigma}$ is a product of generators so it determines an element of $W$, although $\mathbf{w}^{\sigma}$ may not be reduced. For $1 \leq j \leq k$, we also consider initial sequences of masks denoted $\sigma[j]=\left(\sigma_{1}, \ldots, \sigma_{j}\right)$, and the corresponding initial subexpressions $\mathbf{w}^{\sigma[j]}=$ $\mathrm{w}_{1}^{\sigma_{1}} \cdots \mathrm{w}_{j}^{\sigma_{j}}$. In particular, we have $\mathrm{w}^{\sigma[k]}=\mathrm{w}^{\sigma}$. We say the mask $\sigma$ is proper if $\sigma \neq$ $(1,1, \ldots, 1)$.

We say that a position $j$ (for $2 \leq j \leq k$ ) of the fixed reduced expression $\mathrm{w}$ is a defect with respect to the mask $\sigma$ if

$$
l\left(\mathbf{w}^{\sigma[j-1]} \mathbf{w}_{j}\right)<l\left(\mathbf{w}^{\sigma[j-1]}\right) .
$$

Note that the defect status of position $j$ does not depend on the value of $\sigma_{j}$. Let $d_{\mathrm{w}}(\sigma)$ denote the number of defects of w with respect to the mask $\sigma$. We will use the notation $d(\sigma)=d_{\mathrm{w}}(\sigma)$ if the reduced word $\mathrm{w}$ is fixed.

Deodhar's framework gives a combinatorial interpretation for the KazhdanLusztig polynomial $P_{x, w}(q)$ as the generating function for masks $\sigma$ on a reduced expression w with respect to the defect statistic $d(\sigma)$. In this work, we consider the set

$$
\mathcal{S}=\{0,1\}^{l(w)}
$$


of all $2^{l(w)}$ possible masks on w and define a prototype for $P_{x, w}(q)$ :

$$
P_{x}(q)=\sum_{\substack{\sigma \in \mathcal{S} \\ w^{\sigma}=x}} q^{d(\sigma)} .
$$

Definition 2.1 Fix a reduced expression $\mathrm{w}$ for $w$. We say that $\mathcal{S}$ is bounded on $\mathrm{w}$ if $P_{x}(q)$ has degree $\leq \frac{1}{2}(l(w)-l(x)-1)$ for all $x<w$ in Bruhat order.

It is straightforward to show that if $\mathcal{S}$ is bounded on w then it is also bounded on any other reduced expression of $w$.

Theorem 2.2 [3] Let $x, w$ be elements in any Coxeter group $W$, and fix a reduced expression $\mathrm{w}$ for $w$. If $\mathcal{S}$ is bounded on $\mathrm{w}$ then

$$
P_{x, w}(q)=P_{x}(q)=\sum_{\substack{\sigma \in \mathcal{S} \\ \mathrm{w}^{\sigma}=x}} q^{d(\sigma)} .
$$

In [1] and [2], the elements $w$ of finite Weyl groups for which $\mathcal{S}$ is bounded on any (equivalently, every) reduced expression $\mathrm{w}$ of $w$ have been classified using patternavoidance. We call such elements Deodhar. It follows from [2, Corollary 5.3] that all of the Deodhar elements are short-braid avoiding. Here, we say that $w$ is short-braid avoiding if there is no reduced expression for $w$ containing a factor of the form sts where $s$ and $t$ are Coxeter generators satisfying $s t \neq t s$. We refer to the factor $s t s$ as a short-braid. We define an equivalence relation on the set of reduced expressions for a Coxeter element in which two reduced expressions are in the same commutativity class if one can be obtained from the other by a sequence of moves that interchange adjacent Coxeter generators that commute. Recall that by definition, every reduced expression for a fully commutative element can be obtained from any other using commuting moves on the Coxeter generators that appear in the expressions. In the simply laced types $A, D$ and $E, w$ is fully commutative if and only if $w$ is shortbraid avoiding.

By [1, Lemma 2], we have that $\mathcal{S}$ is bounded on w if and only if for every proper mask $\sigma \in \mathcal{S} \backslash\{(1,1, \ldots, 1)\}$, we have

$$
D(\sigma)=\# \text { of plain-zeros of } \sigma-\# \text { of zero-defects of } \sigma>0 \text {. }
$$

We call $D(\sigma)$ the Deodhar statistic of $\sigma$. Here, a position in w is a zero-defect if it has mask-value 0 and it is also a defect. A position in $\mathrm{w}$ is a plain-zero if it has maskvalue 0 and it is not a defect. Similarly, we say that a position with mask-value 1 is a one-defect if it is a defect, and we say that the position is a plain-one otherwise.

\subsection{Heaps}

If $\mathrm{w}=\mathrm{w}_{1} \cdots \mathrm{w}_{k}$ is a reduced expression for a Coxeter element, then following [19] we define a partial ordering on the indices $\{1, \ldots, k\}$ by the transitive closure of the relation $i \lessdot j$ if $i<j$ and $\mathrm{w}_{i}$ does not commute with $\mathrm{w}_{j}$. We label each element $i$ 
of the poset by the corresponding generator $w_{i}$. It follows quickly from the definition that if $w$ and $w^{\prime}$ are two reduced expressions for an element $w$ that are in the same commutativity class then there exists a poset isomorphism $\phi$ such that $\mathrm{w}_{i}=\mathrm{w}_{\phi(i)}^{\prime}$ for all $i \in\{1, \ldots, k\}$. This isomorphism class of labeled posets is called the heap of $\mathrm{w}$, where $\mathrm{w}$ is a reduced expression representative for a commutativity class of $w$. In particular, if $w$ is fully commutative then it has a single commutativity class, and so there is a unique heap that we refer to as the heap of $w$.

Cartier and Foata [20] were among the first to study heaps of dimers, which were generalized to other settings by Viennot [21]. Stembridge has studied enumerative aspects of heaps $[19,22]$ in the context of fully commutative elements. Green has also considered heaps of pieces with applications to Coxeter groups in [23-25].

The Coxeter graph of type $A_{n}$ has the form

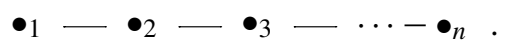

As in [1], we will represent a heap of a permutation as a set of lattice points embedded in $\mathbb{N}^{2}$. To do so, we assign coordinates $(x, y) \in \mathbb{N}^{2}$ to each entry of the labeled Hasse diagram for the heap of $w$ in such a way that:

(1) An entry represented by $(x, y)$ is labeled by the Coxeter generator $s_{i}$ in the heap if and only if $x=i$, and

(2) If an entry represented by $(x, y)$ is greater than an entry represented by $\left(x^{\prime}, y^{\prime}\right)$ in the heap then $y>y^{\prime}$.

Since the Coxeter graph of type $A$ is a path, it follows from the definition that $(x, y)$ covers $\left(x^{\prime}, y^{\prime}\right)$ in the heap if and only if $x=x^{\prime} \pm 1, y>y^{\prime}$, and there are no entries $\left(x^{\prime \prime}, y^{\prime \prime}\right)$ such that $x^{\prime \prime} \in\left\{x, x^{\prime}\right\}$ and $y^{\prime}<y^{\prime \prime}<y$. Hence, we can completely reconstruct the edges of the Hasse diagram and the corresponding heap poset from a lattice point representation. This representation enables us to make arguments "by picture" that would otherwise be difficult to formulate.

Although there are many coordinate assignments for any particular heap, the $x$ coordinates of each entry are fixed for all of them, and the coordinate assignments of any two entries only differs in the amount of vertical space between them. In the case that $w$ is fully commutative, a canonical choice can be made by "coalescing" the entries as in [1].

To carry this out, we form the heap of a reduced expression $w$ by reading $w$ from left to right and dropping a point into the $i$ th column representing each generator $w_{i}$. We envision each point as being slightly wider than its column and under the influence of gravity, in the sense that the point must fall to the lowest possible position in the column over the generator corresponding to $\mathrm{w}_{i}$ without passing any previously placed points in adjacent columns. We say that two points $(x, y),\left(x^{\prime}, y^{\prime}\right)$ are connected if $x=x^{\prime} \pm 1$ and $y=y^{\prime} \pm 1$. Whenever there exist two points in adjacent columns $i, i+1$ with empty lattice points between them in columns $i, i+1$, it follows that the points belong to distinct connected components because $w$ is fully commutative. We then allow component containing the lower point to rise up until it is blocked by the higher point. This has the effect of merging the two components into a single connected component. We apply these elevations until the heap is pushed together as much as possible. 
In the example below, we show the heap of the reduced expression $\mathrm{w}=s_{1} s_{4} s_{2} s_{3} s_{5}$ before and after coalescing.

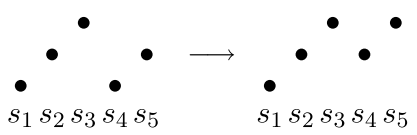

In type $A$, the heap construction can be combined with another combinatorial model for permutations in which the entries from the 1-line notation are represented by strings. Here, the 1 -line notation $w=\left[w_{1} w_{2} \cdots w_{n}\right]$ specifies the permutation $w$ as the bijection mapping $i$ to $w_{i}$. The points at which two strings cross can be viewed as adjacent transpositions of the 1-line notation. Hence, we may overlay strings on top of a heap diagram to recover the 1-line notation for the element by drawing the strings from bottom to top so that they cross at each entry in the heap where they meet and bounce at each lattice point not in the heap. Conversely, each permutation string diagram corresponds with a heap by taking all of the points where the strings cross as the entries of the heap.

For example, we can overlay strings on the two heaps of the permutation [3214] and the heap of $s_{1} s_{4} s_{2} s_{3} s_{5}$. Note that the labels in the picture below refer to the strings, not the generators.

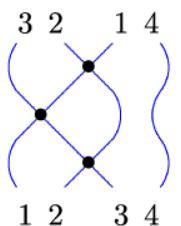

$s_{2} s_{1} s_{2}$

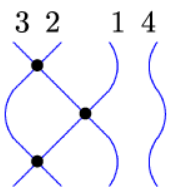

1234

$s_{1} s_{2} s_{1}$

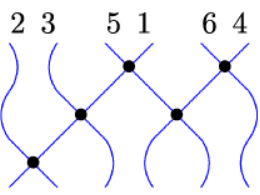

$1234 \quad 56$

$s_{1} s_{4} s_{2} s_{3} s_{5}$

\subsection{Heaps of types $B$ and $D$}

The Coxeter graph of type $B_{n}$ is of the form

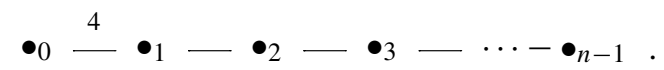

From this graph, we see that the symmetric group $S_{n-1}$ is a parabolic subgroup of this Coxeter group. Because of this, the elements of this group have a standard 1-line notation in which a subset of the entries are barred. We often think of the barred entries as negative numbers, and this group is referred to as the group of signed permutations or the hyperoctahedral group. The action of the generators on the 1-line notation is the same for $\left\{s_{1}, s_{2}, \ldots, s_{n-1}\right\}$ as in type $A$ in which $s_{i}$ interchanges the entries in positions $i$ and $i+1$ in the 1-line notation for $w$. The $s_{0}$ generator acts on the right of $w$ by changing the sign of the first entry in the 1-line notation for $w$. For example, $w=[\overline{4} 2 \overline{3} 1]$ is an element of $B_{4}$ and

$$
s_{0}=[\overline{1} 234]
$$




$$
\begin{aligned}
& w s_{0}=[42 \overline{3} 1] \\
& w s_{1}=[2 \overline{4} \overline{3} 1] .
\end{aligned}
$$

The Coxeter graph for type $D_{n}$ is

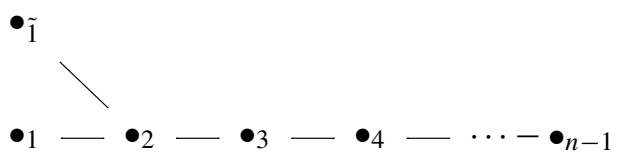

The elements of type $D$ can be viewed as the subgroup of $B_{n}$ consisting of signed permutations with an even number of barred entries. The action of the generators on the 1-line notation is the same for $\left\{s_{1}, s_{2}, \ldots\right\}$ as in type $A$ in which $s_{i}$ interchanges the entries in positions $i$ and $i+1$ in the 1-line notation for $w$. The $s_{\tilde{1}}$ generator acts on the right of $w$ by marking the first two entries in the 1-line notation for $w$ with bars and interchanging them. For example, $w=[\overline{4} 2 \overline{3} 1]$ is an element of $D_{4}$ and

$$
w s_{\tilde{1}}=[\overline{2} 4 \overline{3} 1] .
$$

Although the Coxeter graph for type $D$ has a fork, we will assign coordinates to draw the heap of elements of type $D$ in a linearized way by allowing entries in the first column to consist of either generator $s_{1}, s_{\tilde{1}}$, or both:

$$
\begin{aligned}
s_{1} & =\bullet \\
s_{\tilde{1}} & =\tilde{\bullet} \\
s_{1} s_{\tilde{1}} & =\bullet \tilde{\bullet} .
\end{aligned}
$$

Here we have that $s_{1} s_{\tilde{1}}=s_{\tilde{1}} s_{1}$ so these generators occupy the same level of the heap if there are no non-commuting generators between them. Hence, if $w \in D_{n}$ then the heap of $\mathrm{w}$ has coordinates from $[n-1] \times \mathbb{N}$, where $[n-1]=\{1,2, \ldots, n-1\}$ and lattice points in the first column may be assigned to two entries of the heap.

As in type $A$, we can adorn the heap of w with strings that represent the entries of the 1-line notation for the element. If we label the strings at the bottom of the diagram with the numbers from $1, \ldots, n$ then the $s_{\tilde{1}}$ generator crosses the strings that intersect it and changes the sign on the labels for both strings. All other generators simply cross the strings as in type $A$. For example, the heap of the reduced expression $s_{1} s_{2} s_{3} s_{1} s_{2} s_{1} s_{1}$ is shown below.

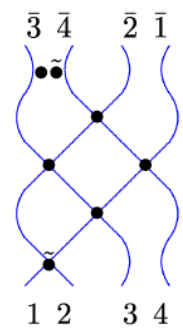


Table 1 Heap decorations

\begin{tabular}{ll}
\hline Decoration & Mask-value \\
\hline$\diamond$ & zero-defect entry \\
plain-zero entry (not a defect) \\
mask-value 1 entry \\
entry of the heap with unknown mask-value \\
lattice point not necessarily in the heap \\
\hline
\end{tabular}

Let $\mathrm{w}$ be a reduced expression for an element $w$ of type $A$ or type $D$. Suppose $x$ and $y$ are a pair of entries in the heap of $w$ that correspond to the same generator $s_{i}$, so they lie in the same column $i$ of the heap. Assume that $x$ and $y$ are a minimal pair in the sense that there is no other entry between them in column $i$. Then, for $w$ to be reduced, there must exist at least one generator that does not commute with $s_{i}$ lying between $x$ and $y$, and if $\mathrm{w}$ is short-braid avoiding, there must actually be two entries that do not commute with $s_{i}$ and lie strictly between $x$ and $y$ in the heap poset. We call two such non-commuting labeled heap entries a resolution of the pair $x, y$. If both of the generators in a resolution lie in column $i-1(i+1$, respectively), we call the resolution a left (right, respectively) resolution. If the generators lie in distinct columns, we call the resolution a distinct resolution. If $w \in D_{n}$ is short braid avoiding and every minimal pair of entries in the heap of $w$ has a distinct resolution then we say $w$ is a convex element. The heap of a convex element in type $D$ can be coalesced just as in type $A$; see [2, Section 7] for details.

Lemma 2.3 (Lateral Convexity Lemma) [1, Lemma 1] If $w$ is a fully commutative permutation then every minimal pair in the coalesced heap of $w$ has a distinct resolution.

For example, lateral convexity implies that if the coalesced heap of a fully commutative permutation contains the points $(2,3)$ and $(2,1)$ then the heap must also contain the points $(1,2)$ and $(3,2)$ as illustrated below.

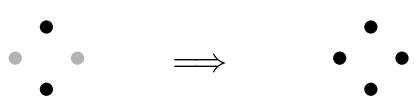

We will frequently interpret a mask applied to a reduced expression for a Deodhar element as a decorated heap diagram, and overlay strings on this decorated heap. In particular, since Deodhar elements are fully commutative, we may refer to a mask $\sigma$ on the element $w$ and draw the unique coalesced decorated heap associated to $\sigma$, rather than explicitly fixing a reduced expression $w$ for each mask we consider. We allow the strings to cross exactly at mask-value 1 entries of the decorated heap so that the strings encode the 1-line notation for $w^{\sigma}$. In the figures that follow, we decorate the heap diagrams according to mask-value using Table 1.

In type $A$, every entry of the decorated heap associated to $\sigma$ has two strings emanating downward from the entry. An entry is a defect precisely when these two strings 
cross below the entry an odd number of times. This follows because an odd number of string crossings below the entry $\mathrm{w}_{j}$ in the decorated heap of $\mathrm{w}$ corresponds to a right descent in the 1-line notation for $\mathrm{w}^{\sigma[j-1]}$. Hence, each string of a defect $d$ in a Deodhar permutation must encounter an entry with mask-value 0 in order for the strings to eventually cross. We call the first such entry along the path of the left (right) string as the left (right, respectively) critical zero of $d$. We denote these critical zeros by $\operatorname{lcz}(d)$ and $\operatorname{rcz}(d)$ respectively, and when $d$ has mask-value 0 then we declare that $d$ is a critical zero of itself as well.

If $d$ has coordinates $(x, y)$ then the left critical zero is the entry $(x-i, y-i)$ where $i>0$ is minimal such that the mask-value of $(x-i, y-i)$ is 0 . Similarly, the right critical zero is the entry $(x+j, y-j)$ where $j>0$ is minimal such that the mask-value of $(x+j, y-j)$ is 0 .

For example, we have $\operatorname{lcz}(d)=p$ and $\operatorname{rcz}(d)=q$ in the heap shown below.

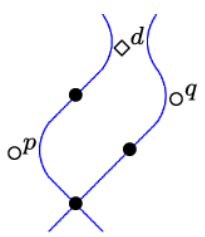

The analogous result using signed strings to determine the defect status of a heap entry in type $D$ is given in [2, Lemma 6.1]. When the entry being tested does not correspond to $s_{\tilde{1}}$, the test is simply the signed version of the inversion test from type $A$.

The following heap fragments called the I-shape and the 4-stack are indicated with black entries below. These fragments were introduced for type $D$ in [2].

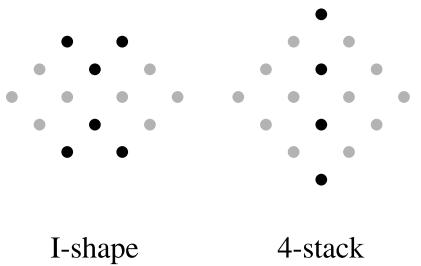

Lemma 2.4 If $w$ is a Deodhar element in type A or a convex Deodhar element of type $D$ with entries from at least two levels in the first column of the heap of $w$, then the heap of $w$ does not contain an I-shape or a 4-stack.

Proof By the Lateral Convexity Lemma 2.3, any heap of type $A$ containing entries in either of the configurations must contain a hexagon and is consequently not Deodhar. This result is extended in [2, Lemma 8.5] to convex elements of type $D$ with entries from at least two levels in the first column.

We say that a 3-stack is the heap fragment obtained from the 4-stack by removing the top or bottom entry but not both. 
$2.4 \mu$-masks for elements of finite Weyl groups

We begin by interpreting $\mu(x, w)$ in Deodhar's framework.

Definition 2.5 Fix a reduced expression $w$ for a Deodhar element $w$ in any Coxeter group. We say that a proper mask $\sigma \in \mathcal{S}$ is a $\mu$-mask for $\mathrm{w}$ if

$$
\# \text { defects of } \sigma=d(\sigma)=\frac{l(\mathrm{w})-l\left(\mathrm{w}^{\sigma}\right)-1}{2} .
$$

A term contributing to the $\mu$ coefficient occurs in degree $\frac{1}{2}\left(l(\mathrm{w})-l\left(\mathrm{w}^{\sigma}\right)-1\right)$ of the Kazhdan-Lusztig polynomial $P_{\mathrm{w}^{\sigma}, \mathrm{w}}(q)$. Hence, by Theorem 2.2 we have

$$
\mu(x, w)=\#\left\{\sigma \in \mathcal{S} \mid \mathrm{w}^{\sigma}=x \text { and } \sigma \text { is a } \mu \text {-mask for } \mathrm{w}\right\}
$$

when $w$ is Deodhar.

Lemma 2.6 Let $\mathrm{w}$ be a reduced expression for a Deodhar element $w$ in any Coxeter group and let $\sigma$ be a mask on $\mathrm{w}$. Then,

$$
l(\mathrm{w})-l\left(\mathrm{w}^{\sigma}\right)-2 d(\sigma)=D(\sigma) .
$$

In particular, $\sigma$ is a $\mu$-mask for $\mathrm{w}$ if and only if

$$
\text { \# of plain-zeros of } \sigma-\# \text { of zero-defects of } \sigma=D(\sigma)=1 \text {. }
$$

The following proof uses a similar technique to [1, Lemma 2].

Proof Observe that

$$
\begin{aligned}
& \frac{1}{2}\left(l(\mathrm{w})-l\left(\mathrm{w}^{\sigma}\right)-1\right)=d(\sigma) \\
& \quad \Longleftrightarrow \quad l(\mathrm{w})-l\left(\mathrm{w}^{\sigma}\right)-2 d(\sigma)=1 .
\end{aligned}
$$

We show that Equation (2.3) holds by induction on the length of w.

One can check that (2.3) holds when $l(\mathrm{w})=1$. Suppose (2.3) holds for all w such that $l(\mathrm{w}) \leq k$. Next, let $\mathrm{w}$ be a reduced expression for a Deodhar element of length $k$ and suppose that $\mathrm{ws}_{i}>\mathrm{w}$, so $\mathrm{w} s_{i}$ is a reduced expression of length $k+1$. Every mask for $w s_{i}$ is obtained from a mask on $w$ extended by 0 or 1 at the last generator. Consider the effect on the left and right sides of (2.3) respectively, in each of the possible cases as shown in Table 2.

By induction, the left and right sides of (2.3) are equal for arbitrary $\mathrm{w}$ and $\sigma$.

It follows from the definition of $P_{x, w}(q)$ that if $l(w)-l(x)=1$ then

$$
\mu(x, w)= \begin{cases}1, & \text { if } x<w \text { in Bruhat order } \\ 0, & \text { otherwise }\end{cases}
$$


Table 2 Multiplication by $s_{i}$

\begin{tabular}{lllc}
\hline Mask-value of $s_{i}$ & Defect-status of $s_{i}$ & Change in left side & Change in right side \\
\hline 1 & not a defect & $1-1=0$ & 0 \\
1 & defect & $1-(-1)-2(1)=0$ & 0 \\
0 & not a defect & 1 & 1 \\
0 & defect & $1-2(1)=-1$ & -1 \\
\hline
\end{tabular}

We say that a $\mu$-mask $\sigma$ on $\mathrm{w}$ is defective if $l(\mathrm{w})-l\left(\mathrm{w}^{\sigma}\right)>1$. It follows directly from Lemma 2.6 that $\sigma$ is a defective $\mu$-mask if and only if $\sigma$ is a $\mu$-mask with at least one defect.

Lemma 2.7 Let $\mathrm{w}$ be a Deodhar element in any Coxeter group and $\sigma$ be a $\mu$-mask on $\mathrm{w}$. If $\sigma^{\prime}$ is any proper mask obtained from $\sigma$ by changing the mask-value of a plain-zero to have mask-value 1 , then $\sigma^{\prime}$ must have strictly fewer zero-defects than $\sigma$. Also, there are no maximal entries in the heap of $\mathrm{w}$ that are one-defects in $\sigma$.

Proof If $\sigma^{\prime}$ does not have fewer zero-defects than $\sigma$ then since $\sigma^{\prime}$ has one fewer plain-zero than $\sigma$ we have

$$
\begin{aligned}
D\left(\sigma^{\prime}\right) & =\text { \# plain-zeros } \sigma^{\prime}-\text { \# zero-defects } \sigma^{\prime} \\
& <\text { \# plain-zeros } \sigma-\text { \# zero-defects } \sigma=D(\sigma)=1
\end{aligned}
$$

contradicting Equation (2.1).

Similarly, if there is a maximal one-defect $d$ in the decorated heap of $\sigma$ then we can form a proper mask $\sigma^{\prime \prime}$ on w by changing the mask-value of $d$ to 0 . Observe that $d$ is a zero-defect in $\sigma^{\prime \prime}$ and every other entry in $\sigma^{\prime \prime}$ has the same defect status as in $\sigma$ since $d$ is heap-maximal. Since $\sigma$ is a $\mu$-mask we have $D(\sigma)=1$ so $D\left(\sigma^{\prime \prime}\right)=0$ which contradicts Equation (2.1).

Lemma 2.8 Let $W$ be a Coxeter group. For any $w, x \in W$ we have $\mu(x, w)=$ $\mu\left(x^{-1}, w^{-1}\right)$.

Proof This follows from the standard fact that $P_{x, w}(q)=P_{x^{-1}, w^{-1}}(q)$; see [5, Chapter 5] for example.

\section{Properties of $\mu$-masks in type $A$}

In this section we restrict to type $A$. Recall from [1] the construction of the defect graph $G_{\sigma}$ associated to $\sigma$. The vertices of $G_{\sigma}$ consist of the zero-defects of $\sigma$, and there exists an edge between two entries $x$ and $y$ if they share any critical zeros:

$$
\{\operatorname{lcz}(x), \operatorname{rcz}(x), x\} \cap\{\operatorname{rcz}(y), \operatorname{lcz}(y), y\} \neq \emptyset .
$$


Example 3.1 Here is a decorated heap corresponding to a typical $\mu$-mask for a Deodhar permutation.

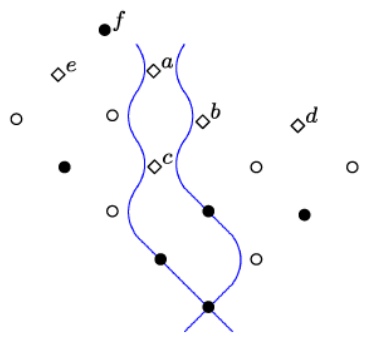

The defect graph for this mask is

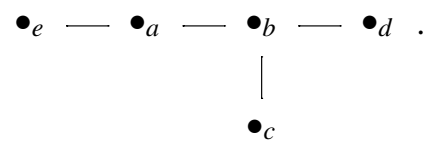

Lemma 3.2 [1, Proposition 1, Lemma 5] Suppose $w$ is a Deodhar permutation and $\sigma$ is any mask on $w$. Then,

(1) The defect graph $G_{\sigma}$ is a forest.

(2) In the decorated heap of $\sigma$, no entry is a critical zero for more than two zerodefects, including itself.

The following result describes the defect graphs of defective $\mu$-masks.

Lemma 3.3 Let $\sigma$ be a mask for a Deodhar permutation $w$. We have that $\sigma$ is a defective $\mu$-mask if and only if $G_{\sigma}$ is a tree with at least one vertex and every plainzero in $\sigma$ is a critical zero for some zero-defect.

Proof Suppose $\sigma$ is a mask for $w$. Every zero-defect has three critical zeros including itself, and no point is a critical zero for more than two zero-defects by Lemma 3.2(2). Hence, the number of critical zeros that are shared by some pair of zero-defects is given by the number of edges in $G_{\sigma}$. Let $V$ and $E$ be the number of vertices and edges in $G_{\sigma}$ respectively. Let $X$ be the number of plain-zeros that are not critical for any zero-defect in $\sigma$. Then we have

$$
\begin{aligned}
& \text { \# plain-zeros of } \sigma+\text { \# zero-defects of } \sigma=3 V-E+X \\
& \text { \# zero-defects in } \sigma=V
\end{aligned}
$$

so,

$$
\begin{aligned}
\text { \# plain-zeros in } \sigma & =2 V-E+X \\
D(\sigma)=\text { \# plain-zeros - \# zero-defects in } \sigma & =V-E+X .
\end{aligned}
$$


If $\sigma$ is a defective $\mu$-mask then by Lemma 2.6 we have

$$
V-E+X=1
$$

Since $\sigma$ is a defective $\mu$-mask, Equation (2.3) implies that $d(\sigma)>0$. If every defect in $\sigma$ is a one-defect then Lemma 2.6 implies that $\sigma$ has a single plain-zero and therefore a single mask-value 0 entry. But every defect requires two critical zeros in the decorated heap of a Deodhar permutation so there must be at least two mask-value 0 entries in $\sigma$, which is a contradiction.

Thus, $G_{\sigma}$ has at least one vertex and by Lemma 3.2(1) we have that $G_{\sigma}$ is a forest. Therefore, $E \leq V-1$. This implies $X \leq 0$ so $X=0$. Furthermore, $E=V-1$ so $G_{\sigma}$ is connected.

On the other hand, if $G_{\sigma}$ is a tree with at least one vertex then $V-E=1$. Also, if every plain-zero is a critical zero for some zero-defect then $X=0$. Hence, we have

$$
D(\sigma)=V-E+X=1
$$

so $\sigma$ is a defective $\mu$-mask by Lemma 2.6.

In the proofs that follow, we will frequently use the fact from Lemma 3.3 that $G_{\sigma}$ is connected if $\sigma$ is a defective $\mu$-mask to contradict the hypothesis that a given decorated heap fragment is actually part of a defective $\mu$-mask.

Example 3.4 Consider the decorated heap fragment shown below associated to a mask $\sigma$ on a Deodhar permutation $w$. Suppose that e corresponds to the Coxeter generator $s_{3}$.

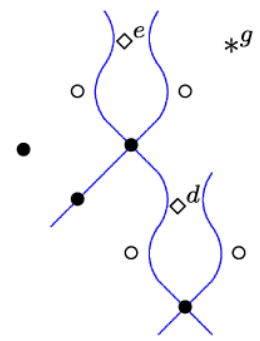

Observe that $g$ is not in the heap by Lemma 2.4 because $g$ would form an Ishape with the other entries. By lateral convexity, the only entries of $\sigma$ corresponding to $s_{i}$ for $i \in\{1,2,3\}$ are the ones already shown in the fragment. Therefore, $\{e\}$ is a connected component of $G_{\sigma}$. Hence, Lemma 3.3 implies that $\sigma$ is not a $\mu$-mask because $d$ and e are disconnected in $G_{\sigma}$ regardless of the mask-value/defect-status of the other entries in $\sigma$ that are not shown.

\section{Proof of the 0-1 property for Deodhar elements in type $A$}

We are now in a position to prove our main result for type $A$. 


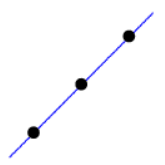

(a)

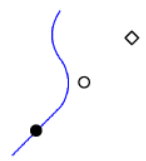

(b)

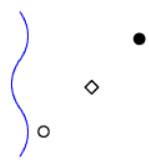

(c)

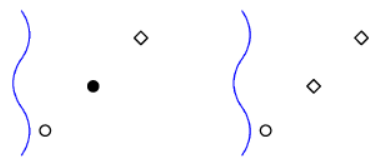

(d)

(e)

Fig. 1 Maximal diagonal of defective $\mu$-masks

Theorem 4.1 Let $w$ be a Deodhar permutation and suppose $x$ is another permutation. Then, $\mu(x, w) \in\{0,1\}$.

Proof Let $w$ be a minimal length element such that $w$ is Deodhar and $\mu(x, w)>1$ for some $x$. Then there exist distinct defective $\mu$-masks $\sigma$ and $\gamma$ such that $w^{\sigma}=x=w^{\gamma}$.

Consider the coalesced heap of $w$. By the Lateral Convexity Lemma 2.3, $w$ has a single entry in the leftmost occupied column of the heap and since $w$ is Deodhar there are fewer than 4 entries in the fourth occupied column by Lemma 2.4. Hence, at least one of the points labeled $y$ or $y^{\prime}$ is not in the heap:

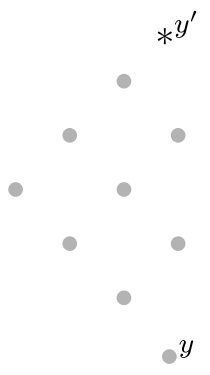

By Lemma 2.8, we may assume that the entry $y^{\prime}$ is not in the heap by taking a distinct pair of $\mu$-masks for $x^{-1}$ on $w^{-1}$ if necessary, because the heap of $w^{-1}$ is obtained from the heap of $w$ by flipping the diagram upside down.

Suppose there are exactly three entries in the maximal northwest diagonal of the heap of $w$ and consider the decorated heap associated to a defective $\mu$-mask on $w$. If any of the entries along the maximal northwest diagonal of the heap of $w$ are a plain-zero then the entry must be the left critical zero of a zero-defect by Lemma 3.3. Hence, the decorated heap of any $\mu$-mask on $w$ has one of the forms shown in Figure 1 on the entries in the maximal northwest diagonal.

Remark 4.2 In the arguments below, we will frequently use the fact that the strings on a decorated heap associated to the mask $\sigma$ encode the 1-line notation for the element $x=w^{\sigma}$. If we label the strings according to their position along the bottom of the decorated heap then the labeled strings must have the same configuration of positions at the top of the decorated heap of $\sigma$ as they do in the decorated heap of $\gamma$ because $w^{\sigma}=x=w^{\gamma}$.

Claim (1): The $\mu$-masks $\sigma$ and $\gamma$ must fall into the same case (a)-(e) of Figure 1. 
Fig. 2 Case (c) distinguished from case (d)

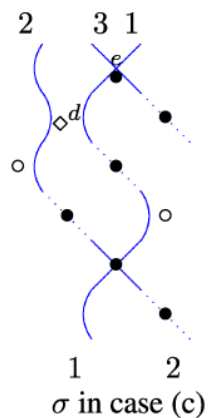

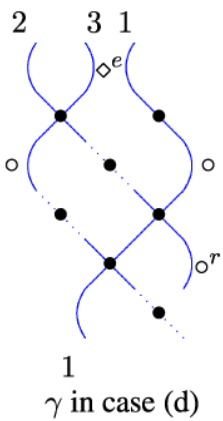

Case: (a) $\Leftrightarrow$ (a). Suppose $\sigma$ falls into case (a) and add strings to the decorated heap associated to $\sigma$. Since there is a single entry in the first column, the string that is leftmost on the bottom of the decorated heap of $\sigma$ will end up in the fourth position at the top of the decorated heap of $\sigma$. Because $w^{\sigma}=w^{\gamma}$ and none of the other cases send the string that is leftmost on the bottom of the decorated heap to the fourth position on the top, we must have that $\sigma$ falls into case (a) if and only if $\gamma$ falls into case (a).

Case: (b) $\Leftrightarrow$ (b). Similarly, if $\sigma$ is in case (b) then the string that is leftmost on the bottom of the decorated heap of $\sigma$ will end up in the second position at the top of the decorated heap of $\sigma$, and we observe that none of the other cases have this feature. Hence, $\sigma$ falls into case (b) if and only if $\gamma$ falls into case (b).

Case: $\sigma$ in case (c), $\gamma$ in case (d). Next, suppose $\sigma$ falls into case (c) and $\gamma$ falls into case (d). Then we have decorated heap fragments of the form shown in Figure 2. First, observe that the string labeled 2 in $\sigma$ cannot encounter a mask-value 0 entry along the minimal diagonal until after it has crossed the string labeled 1 , since otherwise $d$ is not a zero-defect. If the string labeled 3 in $\sigma$ crosses the string labeled 1 below $e$ then $e$ is a maximal one-defect in $\sigma$, contradicting Lemma 2.7. Hence, the string labeled 3 must end up lying to the right of the string labeled 1 on the bottom of the decorated heap of $\gamma$ since $w^{\sigma}=w^{\gamma}$. This implies that the string labeled 3 cannot encounter a mask-value 0 entry along the minimal diagonal in $\gamma$ until after it has crossed the string labeled 1 . Since the string labeled 2 lies to the right of the string labeled 1 on the bottom of the decorated heap of $\sigma$, we have that the string labeled 2 cannot encounter a mask-value 0 entry from the top in $\gamma$ until after it has crossed the string labeled 1. Therefore, the position of the right critical zero of $e$ in $\gamma$ is completely determined by the position of the string labeled 1 on the bottom of the decorated heap of $\sigma$. Furthermore, the string labeled 2 must eventually encounter a mask-value 0 entry $r$ in $\gamma$ so that it lies in the same position on the bottom of the decorated heap of $\gamma$ as it does in $\sigma$, since $w^{\sigma}=w^{\gamma}$. Indeed, lateral convexity implies that if the string labeled 2 does not encounter a mask-value 0 entry in $\gamma$ then the string ends up on the bottom in a column that is strictly right of any column that can be reached by a string traveling along the minimal southwest diagonal of the heap of $w$.

By Lemma 3.3, $r$ is either a left critical zero of a zero-defect $f$ or $r$ is a zero-defect in $\gamma$, and so there exists an entry directly southeast of $r$ to facilitate the string crossing for the zero-defect. Thus, there can be no entry northeast of the right critical zero of $e$, 
Fig. $3 \sigma$ in case (e)

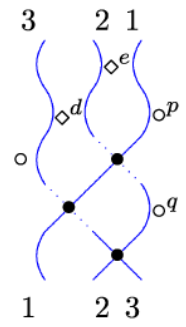

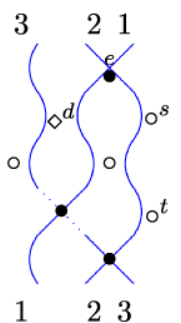

(1)

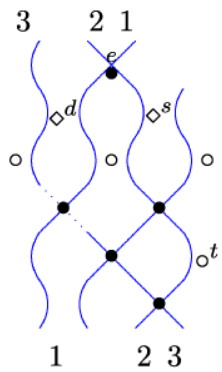

(2)

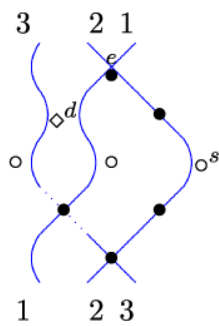

(3)

Fig. $4 \gamma$ in case (c) distinguished from $\sigma$ in case (e)

for otherwise we obtain an I-shape in the heap of $w$, contradicting that $w$ is Deodhar. However, since the right critical zero of $e$ is not a zero-defect in $\gamma$ because the path of the string labeled 1 is prescribed, this implies that $\{e\}$ is a connected component of $G_{\gamma}$. Since $G_{\gamma}$ also contains one of $\{f, r\}$, we contradict that $\gamma$ is a defective $\mu$-mask by Lemma 3.3.

Case: $\sigma$ in case (e), $\gamma$ in case (c). Next, suppose $\sigma$ falls into case (e) as illustrated in Figure 3. Observe that the 1-line notation for $x=w^{\sigma}$ determines the location of the right critical zeros of the zero-defects of $\sigma$. To see this, first observe that the left string of $e$ becomes the right string of $d$ in $\sigma$, and the strings of $e$ must cross above the right critical zero $q$ of $d$. Otherwise we obtain a 4-stack, contradicting that $w$ is Deodhar. Hence, both of the entries along the path of the right string of $e$ have maskvalue 1 below the right critical zero $p$ of $e$. Thus, the position of the string labeled 1 on the bottom of the heap determines the column where $p$ appears. Similarly, the position of the string labeled 2 on the bottom of the heap determines the column where $q$ appears. Since $w^{\sigma}=w^{\gamma}$, these strings must occur in the same positions at the top and bottom of the decorated heap of $\gamma$.

If $\gamma$ falls into case (c) then the decorated heap of $\gamma$ has one of the forms shown in Figure 4. To see this, begin by observing that the string labeled 3 in $\gamma$ must cross both of the strings labeled 1 and 2 since $w^{\sigma}=w^{\gamma}$. Therefore, the string labeled 3 does not encounter a mask-value 0 entry along the minimal diagonal until it crosses both the strings labeled 1 and 2. Hence, the location of the right critical zero of $d$ in $\gamma$ is completely determined by the location of the string labeled 1 on the bottom of the decorated heap of $\sigma$. Also, observe that the string labeled 2 cannot encounter a mask-value 0 entry from the top in $\gamma$ until the column after the right critical zero of 
$d$ appears, for otherwise the string labeled 2 ends up lying to the left of the string labeled 1 on the bottom of the decorated heap of $\gamma$, contradicting that $w^{\sigma}=w^{\gamma}$. Since the string labeled 2 must cross the string labeled 3 in $\gamma$, the string labeled 2 eventually encounters a mask-value 0 entry $s$, and the string labeled 2 either turns once or the string labeled 2 turns three times before crossing the string labeled 3 .

If the string labeled 2 turns three times in the heap before crossing the string labeled 3, and $s$ is a plain-zero, then $\gamma$ has the form shown in Figure 4(1). To verify this, note that if the string labeled 2 does not meet $\operatorname{rcz}(d)$ then it meets another maskvalue 0 entry $u$ that is a critical zero for a zero-defect $g$ by Lemma 3.3. In fact, we either have $u=g$ or $u=\operatorname{rcz}(g)$. In either case, $\operatorname{lcz}(g)$ prevents the string labeled 3 from crossing the string labeled 2 which is a contradiction. Next, observe that $t$ is a plain-zero so there exists a zero-defect with $t$ as its left critical zero, and the entry directly southeast of $t$ lies in the heap of $w$. Also, $s$ must be the left critical zero of a zero-defect by Lemma 3.3, which introduces an I-shape into the heap contradicting that $w$ is Deodhar.

If the string labeled 2 turns three times in the heap before crossing the string labeled 3, and $s$ is a zero-defect, then $\gamma$ has the form shown in Figure 4(2). To see this consider that if $\operatorname{lcz}(s) \neq \operatorname{rcz}(d)$ then $\{d\}$ is a connected component of $G_{\gamma}$ because any zero-defect that shares a critical zero with $d$ would form an I-shape with the other entries. Since $G_{\gamma}$ also contains $s$ we contradict that $\gamma$ is a defective $\mu$-mask by Lemma 3.3. Next, observe that if the right string of $s$ does not cross the left string of $s$ before the third turning point $t$ of the string labeled 2 then the strings of $s$ do not have an opportunity to meet again so $s$ cannot be a zero-defect. Moreover, $t$ is a plain-zero so there exists a zero-defect $f$ with $t$ as its left critical zero, and the entry directly southeast of $t$ lies in the heap of $w$. Also, the right critical zero of $s$ is a plainzero, and $s$ cannot share a critical zero with another zero-defect to the right because this would introduce an I-shape, contradicting that $w$ is Deodhar. Hence, $\{d, s\}$ is a connected component of $G_{\gamma}$. Since $G_{\gamma}$ also contains $f$ we contradict that $\gamma$ is a defective $\mu$-mask by Lemma 3.3.

If the string labeled 2 turns once in the heap before crossing the string labeled 3 then $\gamma$ has the form shown in Figure 4(3). In this case, the right critical zero of $d$ is a plain-zero that is not shared with another zero-defect to the right, because otherwise this would introduce an I-shape into the heap of $w$, contradicting that $w$ is Deodhar. However, $s$ is a plain-zero that must be the left critical zero of a zero-defect $g$ by Lemma 3.3. Hence, $\{d\}$ is a connected component of $G_{\gamma}$. Since $G_{\gamma}$ also contains $g$ we contradict that $\gamma$ is a defective $\mu$-mask by Lemma 3.3.

Thus, we have shown that $\gamma$ lies in case (c) if and only if $\sigma$ lies in case (c).

Case: $\sigma$ in case (e), $\gamma$ in case (d). Finally, if $\gamma$ falls into case (d) and $\sigma$ falls into case (e) then the decorated heap of $\gamma$ has form shown in Figure 5.

Since $w^{\sigma}=w^{\gamma}$, the string labeled 2 in $\gamma$ cannot encounter a mask-value 0 entry along the minimal diagonal until it has crossed the string labeled 1 . Hence, the string labeled 3 cannot encounter a mask-value 0 entry from the top until it has crossed the string labeled 1 . Therefore, the position of the right critical zero $s$ of $e$ is completely determined by the position of the string labeled 1 on the bottom of the decorated heap of $\sigma$. Since the string labeled 3 in $\sigma$ travels along the minimal southwest diagonal in $\sigma$, we have that the string labeled 3 in $\gamma$ must encounter a mask-value 0 entry $t$ in $\gamma$ 
Fig. $5 \gamma$ in case $(d)$

distinguished from $\sigma$ in case (e)

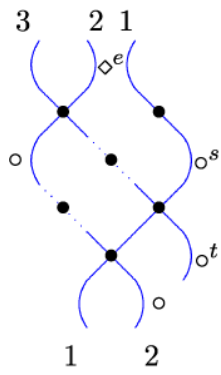

sometime after it has crossed the string labeled 1 . Indeed, lateral convexity implies that if the string labeled 3 does not encounter a mask-value 0 entry in $\gamma$ then the string ends up on the bottom in a column that is strictly right of any column that can be reached by a string traveling along the minimal southwest diagonal of the heap of $w$. The entry $t$ is either a zero-defect or the left critical zero of a zero-defect $h$ by Lemma 3.3, so the entry directly southeast of $t$ lies in the heap of $w$ to facilitate the string crossing for a zero-defect. Hence, there is no entry northeast of $s$, for otherwise we introduce an I-shape into the heap of $w$, contradicting that $w$ is Deodhar. Since the right critical zero of $e$ is a plain-zero, this implies that $e$ does not share a critical zero with any zero-defect lying to the right of $e$. Hence, $\{e\}$ is a connected component of $G_{\gamma}$. Since $G_{\gamma}$ also contains one of $\{t, h\}$ we contradict that $\gamma$ is a defective $\mu$-mask by Lemma 3.3.

Thus, we have shown that $\sigma$ and $\gamma$ fall into the same case from Figure 1, completing the proof of Claim (1).

\section{Claim (2): For each case (a) - (e) of Figure 1, we may construct a smaller length element with distinct $\mu$-masks, contradicting our minimal length choice of $w$. \\ In cases (a), (b), (c) and (d), we can simply remove the three entries on the maximal} northwest diagonal from the decorated heaps of $\sigma$ and $\gamma$. Since $\gamma$ and $\sigma$ fall into the same case, the mask-values agree on these entries. Because the entries are maximal in the decorated heap, removing them does not alter the defect status of any other entries in the decorated heap. Also, in each case we remove the same number of zerodefects as plain-zeros. Hence, we obtain distinct $\mu$-masks $\check{\sigma}$ and $\check{\gamma}$ on a smaller length element $\check{w}$ because $\sigma$ and $\gamma$ were assumed to be distinct. Finally, since $w^{\sigma}=w^{\gamma}$ and the masks agree on the maximal entries that we removed, we have that $\check{w}^{\check{\sigma}}=\check{w}^{\check{\gamma}}$.

Consider the case that $\sigma$ and $\gamma$ are in case (e). Recall from the discussion of Figure 3 that the strings of the zero-defect $e$ always cross above the right critical zero $q$ for the zero-defect $d$, and 1-line notation of $x=w^{\sigma}=w^{\gamma}$ completely determines the location of the right critical zeros $p$ and $q$ of the zero-defects $e$ and $d$. In particular, $p$ and $q$ each occur at the same entry of the heap of $w$ in both $\sigma$ and $\gamma$. If entries exist in the heap of $w$ directly northeast of $p$ and directly southeast of $q$ then we obtain an I-shape in the heap of $w$, contradicting that $w$ is Deodhar.

If $p$ has no entries lying to the northeast then the same strings encounter the entry $p$ from the top in the decorated heaps of both $\sigma$ and $\gamma$. Hence, we may remove $e$ and change the mask-value of $p$ to 1 in $\sigma$ and $\gamma$. This removes one zero-defect 
and one plain-zero from the masks, so we obtain $\mu$-masks $\check{\sigma}$ and $\check{\gamma}$ on a smaller length element $\check{w}$, and the masks are distinct because $\sigma$ and $\gamma$ were assumed to be distinct. Since $w^{\sigma}=w^{\gamma}$ and the same strings encounter $p$ in both masks, we have that $\check{w}^{\check{\sigma}}=\check{w}^{\check{\gamma}}$.

If $q$ has no entries lying to the southeast then the same strings encounter the entry $q$ from the bottom in the decorated heaps of both $\sigma$ and $\gamma$. Also, $d$ does not share a critical-zero with any zero-defect lying to the right. Hence, we may change the mask-value of the left critical zero of $d$ to 1 , and change the mask-value of the right critical zero $q$ of $d$ to 1 in $\sigma$ and $\gamma$. This turns $d$ into a plain-zero, but does not change the defect status of any of the other entries in the decorated heaps. Thus, we obtain masks $\check{\sigma}$ and $\check{\gamma}$ on $w$ where $\check{\sigma}$ and $\check{\gamma}$ fall into case (b) of Figure 1 . Also, $D(\check{\sigma})=D(\sigma)=1=D(\gamma)=D(\check{\gamma})$ so $\check{\sigma}$ and $\check{\gamma}$ are $\mu$-masks. The masks are distinct because $\sigma$ and $\gamma$ were assumed to be distinct. Since $w^{\sigma}=w^{\gamma}$ and the same strings encounter the entries that we changed from the bottom in both masks, we have that $\check{w}^{\breve{\sigma}}=\check{w}^{\check{\gamma}}$. By applying the argument for case (b) above, we can then remove the maximal northwest diagonal of the decorated heaps associated to $\check{\sigma}$ and $\check{\gamma}$ to obtain a smaller length element with distinct $\mu$-masks.

Thus, in all cases we contradict our minimal length choice of $w$. This completes the proof of Claim (2).

By Claim (1) and Claim (2), we obtain a contradiction if there are exactly three entries in the maximal northwest diagonal of the heap of $w$. Hence, we suppose there are fewer than three entries in the maximal northwest diagonal of the heap of $w$. If there is a single entry in the maximal northwest diagonal then any defective $\mu$-mask must have mask-value 1 on this entry by Lemma 3.3. If there are two entries in the maximal northwest diagonal of the decorated heap of $w$ associated to $\sigma$ then either both entries have mask-value 1 , or the maximal entry of the diagonal is a zero-defect and the minimal entry is a plain-zero. Since the leftmost string in the decorated heap corresponds to a fixed point in the permutation $x=w^{\sigma}$ if and only if the maskvalue of the leftmost entry is 0 , we see that the mask-values of the entries in the maximal northwest diagonal agree in the decorated heaps associated to $\sigma$ and $\gamma$. Therefore, we can remove the entries along the maximal northwest diagonal in both decorated heaps. Doing so removes the same number of plain-zeros as zero-defects so the resulting masks are distinct $\mu$-masks on a smaller length element that encode the same element, contradicting our minimal choice of $w$.

Since we obtain a contradiction in every case, we have that $\mu(x, w) \in\{0,1\}$.

Corollary 4.3 If $w$ is a Deodhar element of type $B$ then $\mu(x, w) \in\{0,1\}$ for all $x$.

Proof Let $w$ be a Deodhar element of type B. By [2, Corollary 5.3], the Deodhar elements in type $B$ are short-braid avoiding and an analogue of the Lateral Convexity Lemma 2.3 for type $B$ shows that there can be at most one $s_{0}$ generator in $w$. Therefore, we can interpret any reduced expression for $w$ as a type- $A$ reduced expression by sending the Coxeter generators $s_{0}, s_{1}, \ldots, s_{n-1}$ of type $B$ to the Coxeter generators $s_{1}, s_{2}, \ldots, s_{n}$ of type $A$, even though $\left(s_{0} s_{1}\right)^{3} \neq 1$ in type $B$. This enables us to view the decorated heap of a Deodhar type- $B$ element as the decorated heap of a Deodhar permutation. Moreover, it follows quickly from the discussion in Section 2.3 
that an entry $z$ is a zero-defect in the decorated heap of type $B$ if and only if $z$ is a zero-defect in the corresponding decorated heap of type $A$. In fact, this shows that the Deodhar decorated heaps of type $B_{n}$ are in bijective correspondence with the Deodhar decorated heaps of $A_{n}$ and the bijection preserves the Deodhar statistic. Hence, if there exist distinct $\mu$-masks $\sigma$ and $\gamma$ on $w$ such that $w^{\sigma}=w^{\gamma}$ then the bijection gives a contradiction to Theorem 4.1. Hence $\mu(x, w) \in\{0,1\}$ for all $x$.

This completes the proof of Theorem 1.2 for types $A$ and $B$.

\section{The 0-1 result for Deodhar elements in type $D$}

In this section we sketch the proof of Theorem 1.2 for the Deodhar elements of type $D$ using the methods developed in [2] to characterize the Deodhar elements of type $D$. In particular, we treat the convex and non-convex elements separately. The proof for the convex elements closely follows the proof of our classification result [2, Proposition 8.7] and we cite relevant facts from that proof as necessary.

We begin by recalling a map that is used to obtain a reduction to type $A$.

Lemma 5.1 Let $w$ be a convex Deodhar element of type D and suppose $\sigma$ is a mask on $w$ with three entries in column 1 and no entry directly northeast of the top entry in column 1 . Then, there exists a map $\pi$ that we denote $(w, \sigma) \mapsto(\pi(w), \pi(\sigma))$ with the following properties:

(1) $\pi(w)$ is a Deodhar element of type A.

(2) $\pi(\sigma)$ is a mask on $\pi(w)$.

(3) $D(\pi(\sigma))=D(\sigma)$.

(4) The entries lying to the right of column 3 in the heap of $\pi(w)$ correspond to the entries lying to the right of column 1 in the heap of $w$.Moreover, the maskvalue and defect-status of these entries in decorated heap of $\pi(\sigma)$ agree with the mask-value and defect-status of the corresponding entries in the decorated heap of $\sigma$.

Proof The map of elements is defined in [2, Definition 8.3]. This definition extends to a map of masks as described in Appendix with the given properties by considering the proof of [2, Proposition 8.7].

Proposition 5.2 Suppose $w$ is a convex Deodhar element of type D, and $x$ is any element of type $D$. Then, $\mu(x, w) \in\{0,1\}$.

Proof Suppose for the sake of contradiction that there exist distinct defective $\mu$ masks $\sigma$ and $\gamma$ such that $w^{\sigma}=x=w^{\gamma}$. Let $k(w)$ be the number of distinct occupied levels in the first column of the coalesced heap of $w$. We consider the same cases that were considered in the proof of [2, Proposition 8.7].

Case $\mathbf{k}(\mathbf{w})=\mathbf{0}$ or 1 . If $w$ has at most a single entry in the first column then $w$ is contained in a parabolic subgroup of type $A$. Hence, Theorem 4.1 gives that $\mu(x, w) \in\{0,1\}$ for all $x$. 
Suppose there exist two entries in column 1. These entries correspond to the Coxeter generators $\left\{s_{1}, s_{\tilde{1}}\right\}$. If exactly one of the entries $p$ in column 1 has mask-value 0 in $\sigma$ or $\gamma$ then $p$ cannot be the left critical zero for any zero-defect by [2, Lemma 6.1]. Hence, $p$ contradicts Lemma 2.7. Therefore, both entries in column 1 must have the same mask-value in any $\mu$-mask. Moreover, both entries of column 1 have maskvalue 0 in $\sigma$ if and only if all of the entries in the 1-line notation for $x=w^{\sigma}$ are positive, and this occurs if and only if both entries of column 1 have mask-value 0 in $\gamma$ because $w^{\sigma}=x=w^{\gamma}$. Since $w$ is Deodhar, we may assume by [2, Theorem 8.1] and Lemma 2.8 that there are at most 3 occupied positions along the northeast diagonal of the heap of $w$.

If both entries in column 1 have mask-value 1 then we consider the decorated heaps $\check{\sigma}$ and $\check{\gamma}$ obtained from $\sigma$ and $\gamma$ by removing all of the entries from the northeast diagonal of $w$. It is not possible for the left string of a defect to pass through the entries of the first column. There may be a zero-defect on the northeast diagonal in column 3 but this forces a plain-zero in column 2. Moreover, $\sigma$ has a defect on the northeast diagonal if and only if the string in the second position at the top of the heap is labeled negatively if and only if $\gamma$ has a defect on the northeast diagonal, since $w^{\sigma}=x=w^{\gamma}$. Therefore the mask-values along the northeast diagonal agree in $\sigma$ and $\gamma$ by Lemma 2.7. Hence, $\check{\sigma}$ and $\check{\gamma}$ are distinct defective $\mu$-masks on a Deodhar permutation $\check{w}$ with $\check{w}^{\check{\sigma}}=\check{w}^{\check{\gamma}}$, contradicting Theorem 4.1 .

If both entries $p, q$ in column 1 have mask-value 0 then consider the decorated heaps $\sigma^{\prime}$ and $\gamma^{\prime}$ obtained from $\sigma$ and $\gamma$ by changing the mask-values of $p$ and $q$ to 1 in each heap. By Lemma 2.7 we have that $p$ and $q$ are critical zeros for a zero-defect $d$, so we have removed two plain-zeros and changed $d$ into a plain-zero in $\sigma^{\prime}$ and $\gamma^{\prime}$. Hence, $D\left(\sigma^{\prime}\right)=D(\sigma)=1=D(\gamma)=D\left(\gamma^{\prime}\right)$. Moreover, there exists a zero-defect $e$ such that $d=\operatorname{lcz}(e)$ in $\sigma^{\prime}$ and $\gamma^{\prime}$ by Lemma 2.7. Therefore the mask-values along the northeast diagonal are completely determined, and so they agree in $\sigma$ and $\gamma$. Hence, $w^{\sigma^{\prime}}=w^{\gamma^{\prime}}$ so $\sigma^{\prime}$ and $\gamma^{\prime}$ are distinct defective $\mu$-masks and both entries in column 1 have mask-value 1 , which is a case we previously considered. Hence, we obtain a contradiction in all cases.

Case $\mathbf{k}(\mathbf{w})=2$. By [2, Lemma 6.7], whenever the first column of a type- $D$ heap contains entries on more than one distinct level, we must have that each level of column 1 contains a unique entry, and the entries alternate between the $s_{1}$ and $s_{\tilde{1}}$ generators. Without loss of generality, we can assume the top entry in column 1 of the heap of $w$ is $s_{\tilde{1}}$ since $\mu(x, w)$ is invariant under applying a Coxeter graph automorphism to $x$ and $w$. Since $w$ is Deodhar, we have by Lemma 2.4 that there are at most 3 entries in column 3. By considering $w^{-1}$ if necessary and applying Lemma 2.8 , we can further assume that the maximal northwest diagonal from the top entry in column 1 contains at most two entries:

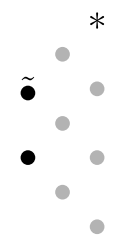


Fig. $6 \mu$-masks with two levels in column 1 $\tilde{0}$

(a) (b)

Consider the decorated heap associated to a $\mu$-mask on $w$. The maximal northwest diagonal of any defective $\mu$-mask on $w$ has one of the forms shown in Figure 6 by Lemma 2.7.

Observe that some strings are labeled negatively in case (b) but all strings are labeled positively in case (a). Since $w^{\sigma}=x=w^{\gamma}$, we have that both masks must fall into the same case, so the mask-values of $\sigma$ and $\gamma$ agree along the maximal northwest diagonal. As in the proof of [2, Proposition 8.7], we may remove this northwest diagonal from $\sigma$ and $\gamma$ to obtain a pair of decorated heaps $\check{\sigma}$ and $\check{\gamma}$ on a Deodhar type- $A$ element $\check{w}$. Observe that $\check{w}^{\check{\sigma}}=\check{w}^{\check{\gamma}}$ and $D(\check{\sigma})=D(\check{\gamma})$. Since $\sigma$ and $\gamma$ are distinct, we obtain a contradiction to Theorem 4.1.

Case $\mathbf{k}(\mathbf{w})=3$. Suppose that $w$ has entries on 3 distinct levels in the first column and assume without loss of generality that the top entry corresponds to $s_{1}$. As in the proof of [2, Proposition 8.7], our strategy will be to project the heap of $w$ to a Deodhar permutation by adding the three entries marked as $\star$ to the left of the heap of $w$ :

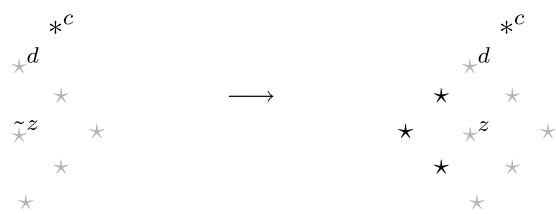

If $w$ contains a 4-stack in column 2 then $w$ is not Deodhar by Lemma 2.4. Hence, we can assume there are at most 3 entries in column 2. By considering $w^{-1}$ if necessary and applying Lemma 2.8, we can assume that the point marked $c$ in column 2 is not in the heap.

Consider the decorated heap associated to a defective $\mu$-mask on $w$. By Lemma 2.7 every plain-zero must be associated with a zero-defect and there are no maximal onedefects. Hence, any defective $\mu$-mask on $w$ has one of the following forms:

(a) $d$ is a plain-one.

(b) $d$ is a zero-defect and $z$ has mask-value 1 .

(c) $d$ is a zero-defect and $z$ has mask-value 0 . The strings for $d$ meet at $z$.

(d) $d$ is a zero-defect and $z$ has mask-value 0 . The strings for $d$ do not meet at $z$.

Our first goal is to show that the $\mu$-masks $\sigma$ and $\gamma$ must fall into the same case of (a)-(d).

Begin by observing that we cannot have $\sigma$ in case (b), and $\gamma$ in case (c) or (d). This follows because $w^{\sigma}=x=w^{\gamma}$ and $x$ has some negatively signed entries in case (b), but $x$ has all positively signed entries in cases (c) and (d).

Next, suppose that $\sigma$ is in case (b) and $\gamma$ is in case (a). Then, we have the decorated heap fragments shown in Figure 7.

The justification for Figure 7 runs as follows. In case (b), the left string of $d$ must become negative as it passes through the $s_{\tilde{1}}$ entry. If we label the string of $d$ that is leftmost on the bottom of the decorated heap by 1, and we label the string of $d$ that is 
Fig. $7 \mu$-masks with three levels in column 1

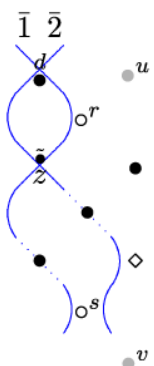

12

$\gamma$ in case (a)

Fig. $8 \mu$-masks with three levels in column 1

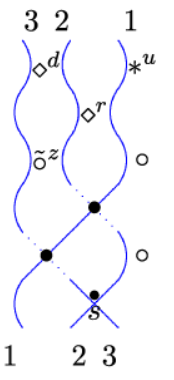

$\sigma$ in case (c)

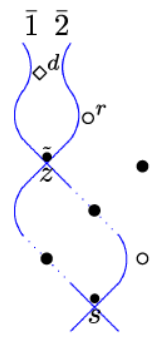

12

$\sigma$ in case (b)

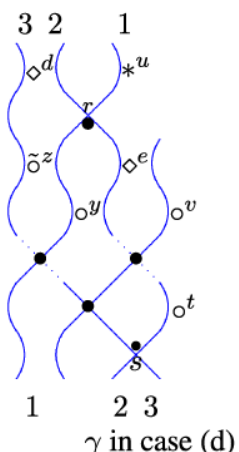

rightmost on the bottom of the decorated heap by 2 , then in order for $d$ to be a defect the strings of $d$ must be labeled at the top by $1 \overline{2}$. This forces the right string of $d$ to also pass through the $s_{\tilde{1}}$ entry so the right critical zero $r$ of $d$ lies next to $d$ as shown. Moreover, the string labeled 2 does not encounter a mask-value 0 entry below $z$ in $\sigma$ until it crosses the string labeled 1. Since $w^{\gamma}=w^{\sigma}$, we must have these strings labeled in the same way in $\gamma$. Hence, the entry $r$ has mask-value 0 in $\gamma$. The entry $r$ is not a zero-defect because the left string of $r$ is negatively labeled while the right string of $r$ is positively labeled. Also, the string labeled 1 must encounter a mask-value 0 entry at $s$ in $\gamma$ in order for the strings of $d$ to be labeled in $\gamma$ as they are in $\sigma$. The entry $s$ is a plain-zero in $\gamma$, for otherwise we introduce a 4-stack into the heap, which contradicts that $w$ is Deodhar. Therefore, there must be zero-defects that have $r$ and $s$ as left critical zeros by Lemma 2.7. But this implies that $u$ and $v$ appear in the decorated heap of $\gamma$, which introduces an I-shape into the heap of $w$, contradicting that $w$ is Deodhar. Thus, we have shown that $\sigma$ is in case (b) if and only if $\gamma$ is in case (b).

Next, suppose that $\sigma$ is in case (c) and $\gamma$ is in case (d). Then, we have the decorated heap fragments shown in Figure 8. In case (c), observe that the right critical zero $r$ for $d$ must lie next to $d$ as shown in order for the strings of $d$ to meet at $z$. Also, the string labeled 3 cannot encounter a mask-value 0 entry below $z$ in $\sigma$ until it crosses the string labeled 2 at some point $s$ on the minimal southwest diagonal because $d$ is a defect. Note that $z$ is a plain-zero as there is no other $s_{\tilde{1}}$ generator in the heap of $w$ to support a defect at $z$, and if we change the mask-value of $z$ to 1 then $d$ remains a zero- 
defect. Hence, $r$ must be a zero-defect with $z$ as its left critical zero by Lemma 2.7. The right string of $d$ becomes the left string of $r$ and the strings of $r$ must cross above the point directly northeast of $s$. Otherwise the strings of $r$ will not have an opportunity to cross again. Hence, $s$ occurs in column 2 or further to the right, and so $u$ is not in the heap as it introduces an I-shape.

Observe that the string that is in the third position at the top of $\sigma$ ends up on the bottom of $\sigma$ in a position lying to the left of the strings labeled 2 and 3. Since $w^{\sigma}=w^{\gamma}$ the strings in $\gamma$ must have the same positions at the top and bottom of the decorated heap as in $\sigma$. The string labeled 3 cannot encounter a mask-value 0 entry below $z$ in $\gamma$ until it crosses the string labeled 2. Since the strings of $d$ do not meet at $z$ in $\gamma$, we have that the entry $r$ must have mask-value 1 . Hence, the only way for the string that is in the third position at the top of $\gamma$ to end up on the bottom of $\gamma$ in a position lying to the left of the strings labeled 2 and 3 is if $\gamma$ has a plain-zero $y$ as shown in Figure 8. The position of the plain-zero $y$ in $\gamma$ is completely determined by the position of the string labeled 1 in $\sigma$ since $w^{\sigma}=w^{\gamma}$. By Lemma 2.7 there exists a zero-defect $e$ with $y$ as its left critical zero. If $e$ lies further from $y$ than shown, we obtain an I-shape in $\gamma$ after taking account of the necessary string crossings for $d$ and $e$. Hence, the right string of $d$ becomes the left string of $e$, and the position of $t$ in $\gamma$ is completely determined by the position of the string labeled 2 in $\sigma$. Also, the right critical zero $v$ of $e$ occurs in a column weakly left of the column of $t$, for otherwise the strings of $e$ will not have an opportunity to cross again.

Observe that $y, t$ and $v$ must be plain-zeros since if any of these entries were zerodefects, the string crossing would introduce a 4-stack into the heap, contradicting that $w$ is Deodhar. By Lemma 2.7, there exists a zero-defect $f$ with $t$ as the left critical zero. Using Lemma 5.1, we can project $\gamma$ to type $A$ and obtain a $\mu$-mask on a Deodhar permutation. Moreover, the projection does not alter the mask-values of the entries to the right of column 1. Consequently, if the zero-defect $e$ does not share a critical zero with another zero-defect to the right, then $e$ and $f$ would be disconnected in the defect graph of the projection of $\gamma$, which is a contradiction. But then we obtain an I-shape in the heap of $w$, contradicting that $w$ is Deodhar.

Finally, it remains to show that if $\sigma$ is in case (c) or (d) then $\gamma$ cannot fall into case (a). If $\gamma$ is in case (a) then $\gamma$ has a decorated heap of the form shown in Figure 9. Since $w^{\sigma}=w^{\gamma}$ we have that there are no negatively labeled strings in $\gamma$ so $z$ must be a plain-zero. Also, the labeled strings at $d$ must form an inversion in the 1-line notation for $x=w^{\sigma}$. Thus, the path of the left string of $d$ must encounter a plain-zero $s$ along the minimal southwest diagonal in order for the strings of $d$ to be labeled in $\gamma$ as they are in $\sigma$. Since $s$ is a plain-zero, we have by Lemma 2.7 that there exists a zerodefect $e$ with $s$ as its left critical zero. Hence, $u$ cannot be in the heap as it would form an I-shape with the string crossing for $e$ by lateral convexity. Since $z$ is a plain-zero, we have by Lemma 2.7 that $r$ must be a zero-defect with $z$ as the left critical zero.

Because $d$ is a zero-defect in $\sigma$, we have that the labeled strings lying in the first and second position at the top of the decorated heap of $\sigma$ form an inversion in the 1-line notation for $x=w^{\sigma}$. Since $r$ is a zero-defect in $\gamma$, the labeled strings lying in the first and third position at the top of the decorated heap of $\gamma$ form an inversion in the 1-line notation for $x=w^{\gamma}$. Hence, the labeled strings in the first three positions at the top of the decorated heaps of both $\sigma$ and $\gamma$ either form a [321] permutation pattern, or a [312] permutation pattern. 
Fig. $9 \gamma$ in case (a)

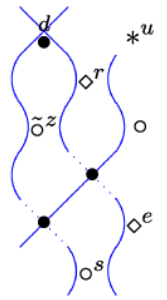

If the labeled strings in the first three positions at the top of the decorated heaps form a [321] pattern then the decorated heap of $\gamma$ has the form shown in Figure 10(1). In particular, the string labeled 1 ends up left of $s$ on the bottom of $\gamma$. Also, neither of the strings labeled 2 or 3 can encounter a mask-value 0 entry below $z$ until after crossing the string labeled 1 in $\gamma$. Hence, the right critical zero of $r$ must be a plain-zero. Using Lemma 5.1, we can project $\gamma$ to type $A$ and obtain a $\mu$-mask on a Deodhar permutation. Moreover, the projection does not alter the mask-values of the entries to the right of column 1. Consequently, if the zero-defect $r$ does not share its right critical zero with another zero-defect, then $e$ and $r$ would be disconnected in the defect graph of the projection of $\gamma$, which is a contradiction. But then we obtain an I-shape in the heap of $w$, contradicting that $w$ is Deodhar.

Suppose the labeled strings in the first three positions at the top of the decorated heaps form a [312] pattern. By the remarks justifying Figure 8, we find that $\sigma$ cannot be in case (c) so $\sigma$ must be in case (d). Hence, the decorated heap of $\sigma$ has the form shown in Figure 10(2). The string labeled 3 cannot encounter a mask-value 0 entry below $z$ in $\sigma$ until it crosses the strings labeled 1 and 2. Hence, the position of $q$ in $\sigma$ is completely determined by the position of the string labeled 2 on the bottom of $\gamma$. Thus, the position of the string labeled 1 in $\gamma$ determines the location of the right critical zero $p$ of $d$ in $\sigma$ and $p$ occurs in a column weakly left of the column of $q$. Since $q$ must be a plain-zero, we have that there exists a zero-defect $f$ northeast of $q$ by Lemma 2.7. Using Lemma 5.1, we can project $\sigma$ to type $A$ and obtain a $\mu$-mask on a Deodhar permutation. Moreover, the projection does not alter the mask-values of the entries to the right of column 1. Consequently, if the zero-defect $d$ does not share its plain right critical zero $p$ with another zero-defect, then $d$ and $f$ would be disconnected in the defect graph of the projection of $\sigma$, which is a contradiction. But then we obtain an I-shape in the heap of $w$, contradicting that $w$ is Deodhar.

Thus, we have shown that $\sigma$ and $\gamma$ always fall into the same case (a) - (d). If $\sigma$ and $\gamma$ fall into case (a) then we can remove the maximal entry $d$ which is a plainone in both masks, and then apply the argument from the case $k(w)=2$ to obtain a contradiction.

Next, assume that $\sigma$ and $\gamma$ fall into case (b), (c) or (d). We apply Lemma 5.1 to add three new entries to the decorated heaps of $\sigma$ and $\gamma$, obtaining a pair of decorated heaps $\check{\sigma}$ and $\check{\gamma}$ on the Deodhar permutation $\pi(w)$. The mask-values of the three new entries depend on which case (b), (c) or (d) the masks fall into, but in each case the projection satisfies $D(\check{\sigma})=D(\check{\gamma})$. To verify that $\pi(w)^{\check{\sigma}}=\pi(w)^{\check{\gamma}}$ we must either show that the same strings encounter the three new entries from the top of the decorated heap of $\sigma$ as in $\gamma$, or we must show that the same strings encounter the three new entries from the bottom of the decorated heap of $\sigma$ as in $\gamma$. 
Fig. $10 \gamma$ in case (a)

distinguished from $\sigma$ in case (c) or (d)

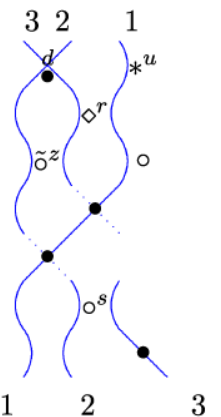

(1) $\gamma$ in case (a)

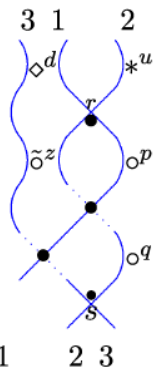

(2) $\sigma$ in case (d)

Consider the heap of $w$ with entries labeled as follows.

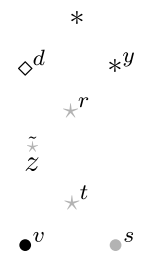

If both $s$ and $y$ lie in the heap of $w$ then we introduce an I-shape, contradicting that $w$ is Deodhar. Hence, at least one of $\{s, y\}$ is not in the heap of $w$. If $s$ is not in the heap of $w$ then the mask-value of $t$ and $r$ in $\sigma$ and $\gamma$ is completely determined by the case (b)-(d) that $\sigma$ and $\gamma$ fall into and the requirement that the strings of $d$ cross at $v$. In particular, the strings encountering $v, t$, and $z$ from the bottom of the decorated heap of $\sigma$ must be the same as the strings encountering $v, t$, and $z$ from the bottom of the decorated heap of $\gamma$. Hence, $\pi(w)^{\check{\sigma}}=\pi(w)^{\check{\gamma}}$ in this case.

On the other hand, if $y$ is not in the heap of $w$ then we may observe that the mask values of $d, z$ and $r$ in $\sigma$ and $\gamma$ are completely determined by the case (b)-(d) that $\sigma$ and $\gamma$ fall into. Thus, we have that the strings encountering $d, r$ and $z$ from the top of the decorated heap of $\sigma$ must be the same as the strings encountering $d, r$ and $z$ from the top of the decorated heap of $\gamma$. Hence, $\pi(w)^{\check{\sigma}}=\pi(w)^{\check{\gamma}}$ in this case.

Since $\sigma$ and $\gamma$ are distinct, we obtain a contradiction to Theorem 4.1.

Case $\mathbf{k}(\mathbf{w})=4$. Suppose that $w$ has entries on 4 distinct levels in the first column, and consider the decorated heap on $w$ that is associated to the defective $\mu$-mask $\sigma$. Following the argument in the proof of [2, Proposition 8.7], we reduce to the case where $\sigma$ contains a decorated heap fragment of the form shown in Figure 11.

If there is a point at $y$ or $y^{\prime}$ then there is a 4-stack in the second column. If there is a point at $u$ or $u^{\prime}$, we obtain an I-shape with the other entries that exist by convexity. In either case, we contradict that $w$ is Deodhar. Similarly, if both $v$ and $v^{\prime}$ exist, then we obtain an I-shape, so at least one of them is not in the heap. By considering $w^{-1}$ if necessary, we can assume that $v^{\prime}$ is not in the heap.

By Lemma 2.7, we can assume that $z$ has mask-value 1 and that it is a string 
Fig. 11 A $\mu$-mask with four levels in column 1

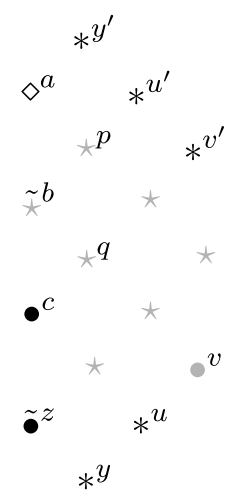

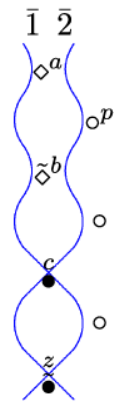

12

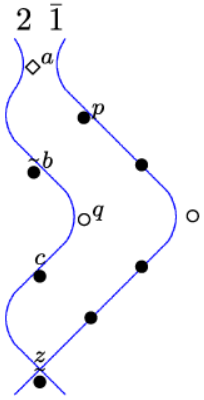

12

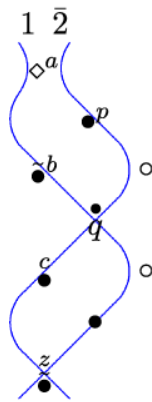

12
21

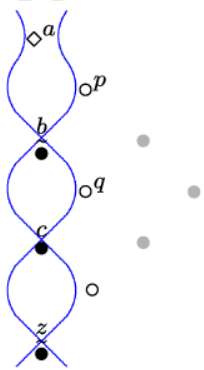

12

(a)

(b)

(c)

(d)

Fig. $12 \sigma$ is a $\mu$-mask with four levels in column 1

crossing for some zero-defect, since if $z$ has mask-value 0 then it can play no critical role in any defect. Also by Lemma 2.7, we have that $a$ is never a plain-zero in any defective $\mu$-mask. Thus, if $a$ is not a zero-defect in at least one of $\{\sigma, \gamma\}$ then it has mask-value 1 in both masks so we can remove it from both masks and obtain a contradiction by considering the $k(w)=3$ case. Therefore, we assume without loss of generality that $a$ is a zero-defect in $\sigma$. This forces $c$ to have mask-value 1 since $a$ cannot be a defect otherwise. Thus, we have a heap fragment of the form in Figure 11.

Also, if $b$ is a plain-zero then $z$ cannot be the string crossing of any zero-defect, regardless of the mask-value for $a$. Hence, we may assume that $b$ is either a zerodefect or has mask-value 1 in all defective $\mu$-masks on $w$.

Working through all of the cases given in the proof of [2, Proposition 8.7] and applying Lemma 2.7, we find that $\sigma$ must have one of the decorated heap fragments shown in Figure 12. For example, the mask-value of $q$ in (c) must be 1, for otherwise $q$ would be a plain-zero that is not associated with any zero-defect. Note that since the labeled strings are distinct among these four cases and $w^{\sigma}=x=w^{\gamma}$, we have that $\sigma$ falls into one of these cases if and only if $\gamma$ falls into the same case. 


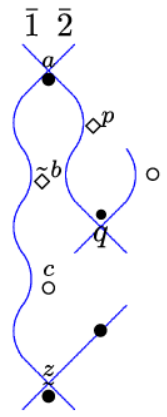

12

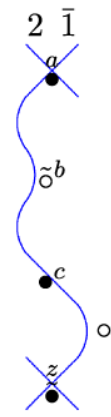

12
$1 \overline{2}$

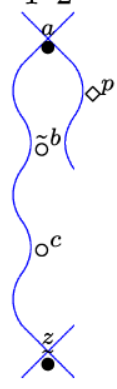

12
21

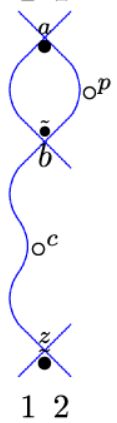

(d)

(a)

(b)

(c)

Fig. $13 \gamma$ is a $\mu$-mask with four levels in column 1

Next, suppose that $\gamma$ has $a$ with mask-value 1 and $\sigma$ falls into one of the cases $\{$ (a), (b), (c), (d) \}. Then we claim $\gamma$ has one of the forms shown in Figure 13, corresponding to the case that $\sigma$ falls into. In any $\mu$-mask we have that $z$ is a plainone. Since $w^{\sigma}=x=w^{\gamma}$, the string paths in $\gamma$ determine the mask-values of the entries in column 1 . In case (a), we have $b$ with mask-value 0 in order that the strings of $a$ be labeled negatively. Hence, $b$ must be a zero-defect whose strings cross at $z$. This implies that the strings of $a$ become the strings of $b$, so by Lemma 2.7 there exists a zero-defect $p$ as shown. Hence, $s_{2}$ is a right descent for the element $x s_{1}$. But this is a contradiction because the form of $\sigma$ shown in Figure 12(a) shows that $s_{2}$ is a right ascent for the same element $x s_{1}$.

In case (b), the string labeled 1 forces $b$ to have mask-value 0 , but then the other string of $a$ must be labeled negatively, contradicting that $w^{\sigma}=x=w^{\gamma}$. Similarly, in case (c), the string labeled 2 forces $b$ to have mask-value 0 , but then the other string of $a$ must be labeled negatively, contradicting that $w^{\sigma}=x=w^{\gamma}$. In case (d), note that $p$ cannot be a defect because the first and last opportunity for the strings of $p$ to cross occurs at $z$ which contradicts that the strings through $a$ are assumed to cross at $z$. But then $p$ is a plain-zero which contradicts Lemma 2.7.

Thus, we have shown that $\sigma$ and $\gamma$ always fall into the same case. As in the proof of [2, Proposition 8.7], we may remove the zero-defect $a$ and change the mask-value of some plain-zero entry to 1 . Specifically, in cases (a), (c) and (d), we change the mask-value of the right critical zero for $a$. Since the entry directly northeast of the right critical zero of $a$ is not in the heap for any of these cases, the same strings encounter the entry from the top of the decorated heap of $\sigma$ as in $\gamma$. In case (b), we change the mask-value of $q$ to have mask-value 1 . Since there is no entry two units northeast from $q$ and the mask-value of the entry directly northeast of $q$ is determined, the same strings encounter $q$ from the top of the decorated heap of $\sigma$ as in $\gamma$.

We obtain in this way a pair of decorated heaps $\check{\sigma}$ and $\check{\gamma}$ on a Deodhar element $\check{w}$ with $k(w)=3$. These reductions remove a single zero-defect and a single plain-zero in each case, so $D(\check{\sigma})=D(\check{\gamma})$. Because $w^{\sigma}=w^{\gamma}$ and the same strings encounter the entries that we changed in both masks we have $\check{w}^{\check{\sigma}}=\breve{w}^{\check{\gamma}}$. Thus, we may apply the argument from the case $k(w)=3$ to obtain a contradiction to Theorem 4.1. 
Fig. 14 Fragment from a non-convex element

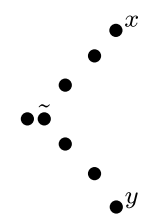

Case $\mathbf{k}(\mathbf{w}) \geq \mathbf{5}$. If $w$ has entries on 5 or more distinct levels in the first column then $w$ is not Deodhar by Lemma 2.4 .

This exhausts the cases, completing the proof of the proposition.

Proposition 5.3 Suppose that $w \in D_{n}$ is a Deodhar element that is not convex. Then, $\mu(x, w) \in\{0,1\}$ for all $x<w$.

Proof Since $w$ is not convex, there exists a minimal pair of entries in column $i \geq 2$ with only a left resolution. By [1, Lemma 6.8], the part of the heap to the left of column $i$ has the particular form shown in Figure 14 in which each column $1, \ldots, i$ has exactly two entries.

Suppose for the sake of contradiction that there exist distinct defective $\mu$-masks $\sigma$ and $\gamma$ such that $w^{\sigma}=x=w^{\gamma}$. If exactly one of the entries in column 1 has maskvalue 0 in $\sigma$ or $\gamma$, then the mask-value 0 entry cannot be the left critical zero for any zero-defect, and this would contradict Lemma 2.7. Hence, both entries in column 1 must have the same mask-value in any $\mu$-mask. Then, both entries of column 1 have mask-value 0 in $\sigma$ if and only if all of the entries in the 1-line notation for $x=w^{\sigma}$ are positive, and this occurs if and only if both entries of column 1 have mask-value 0 in $\gamma$ because $w^{\sigma}=x=w^{\gamma}$. Thus, the mask-values of $\sigma$ and $\gamma$ must agree on the entries in column 1 .

If the lower entry of column $j \leq i$ in the heap fragment has mask-value 0 , then since it cannot be a defect, it must enable the string crossing for some zero-defect lying to the right of column $j$ by Lemma 2.7. In particular, by the argument given in the proof of [2, Lemma 6.10], the top entry of column $j$ must also be a plain-zero.

Observe that for each $j \leq i$ we have that a string in position $j \leq i$ on the bottom of the heap ends up in position $k>j$ at the top of the heap if and only if the bottom entry of column $j$ has mask-value 1 and the top entry of column $j$ has mask-value 0 . Therefore, column $j$ satisfies this condition in $\sigma$ if and only if column $j$ satisfies this condition in $\gamma$ for each $1 \leq j \leq i$ because $w^{\sigma}=x=w^{\gamma}$.

Also, we have that column $j \leq i$ in the heap fragment has both entries with maskvalue 1 if and only if position $j$ is a fixed point in the 1-line notation for $x$, viewing $x$ as a signed permutation. To see this, suppose for the sake of contradiction that $\sigma$ is a defective $\mu$-mask such that $x=w^{\sigma}$ has a fixed point in position $j \leq i$ of the 1-line notation but column $j$ has both entries with mask-value 0 . Then there exists a plain-zero that is not connected to any zero-defect contradicting Lemma 2.7. Hence, column $j$ has both entries with mask-value 1 in $\sigma$ if and only if column $j$ has both entries with mask-value 1 in $\gamma$, for each $1 \leq j \leq i$, because $w^{\sigma}=x=w^{\gamma}$.

Thus, the mask-values of $\sigma$ and $\gamma$ must agree in every column $1, \ldots, i$. The proof of [1, Lemma 6.10] describes how to project any mask $\sigma$ on $w$ to a mask $\check{\sigma}$ on a 
convex element $\check{w}$ obtained from $w$ by removing columns $2, \ldots, i$. This projection has the properties that:

(1) $D(\sigma) \geq D(\check{\sigma})$.

(2) The mask-values of the entries to the right of column $i$ in the decorated heap of $\sigma$ are unchanged in the decorated heap of $\check{\sigma}$.

Since $D(\sigma)=1$ we have by (1) that $D(\check{\sigma}) \leq 1$. If $D(\check{\sigma}) \leq 0$ then either $\check{w}$ is not Deodhar or $\check{\sigma}=(1,1, \ldots, 1)$. If $\check{w}$ is not Deodhar then $w$ is not Deodhar by the proof of [2, Theorem 9.1] which is a contradiction. Hence, we may assume that $\check{\sigma}$ is a $\mu$-mask on $\check{w}$ or that $D(\check{\sigma})=0$ in which case $\check{\sigma}=(1,1, \ldots, 1)$.

Since the mask-values of $\sigma$ and $\gamma$ agree in every column $1, \ldots, i$, we have that $D(\check{\sigma})=D(\check{\gamma})$. If $D(\check{\sigma})=0=D(\check{\gamma})$ then $\check{\sigma}=(1,1, \ldots, 1)=\check{\gamma}$ so $\sigma=\gamma$ which is a contradiction. Hence, $\check{\sigma}$ and $\check{\gamma}$ are distinct $\mu$-masks on the convex element $\check{w}$. Since $w^{\sigma}=w^{\gamma}$ we also have that $\check{w}^{\check{\sigma}}=\check{w}^{\check{\gamma}}$. Because $\sigma$ and $\gamma$ are distinct, we obtain a contradiction to Proposition 5.2.

Combining Propositions 5.2 and 5.3, we have the following result.

Theorem 5.4 Let $w$ be a Deodhar element of type $D$ and suppose $x$ is another element of type $D$. Then, $\mu(x, w) \in\{0,1\}$.

\section{The 0-1 result for Deodhar elements of finite exceptional types}

Theorem 6.1 If $w$ is a Deodhar in type $E_{6}, E_{7}, E_{8}, F_{4}$ or $G_{2}$ then $\mu(x, w) \in\{0,1\}$ for all $x$.

Proof These groups are finite, so the computation of $\mu$ values is verifiable by computer. Some $\mathrm{C}++$ code is available at http://www.math.ucdavis.edu/ brant/code/ for this purpose.

This completes the proof of Theorem 1.2.

Acknowledgements We thank Sara Billey, Richard Green, Monty McGovern, Yuval Roichman, Monica Vazirani and Greg Warrington for many useful conversations and suggestions.

\section{Appendix}

Here is an explicit description of the map defined in Lemma 5.1 that takes a decorated heap of type $D$ to a decorated heap of type $A$. The map adds three new entries to the left of the first column of the type- $D$ heap so that the result is a fully commutative heap in type $A$. The table below indicates the mask-values that should be assigned to these new entries and the entries from the first column of the type- $D$ heap so that the Deodhar statistic is preserved. This assignment of mask-values depends on 
the cases given in the first column. Further details are given in [2, Proposition 8.7].

\begin{tabular}{|l|l|l|}
\hline Case & $(w, \sigma)$ \\
\hline$d$ is a plain-one. & & \\
\hline $\begin{array}{l}d \text { is a zero-defect and } z \\
\text { has mask-value } 1 .\end{array}$ & & \\
\hline $\begin{array}{l}d \text { is a zero-defect and } z \text { has } \\
\text { mask-value } 0 \text {. The strings for } \\
d \text { meet at } z \text {. }\end{array}$
\end{tabular}

\section{References}

1. Billey, S., Warrington, G.S.: Kazhdan-Lusztig polynomials for 321-hexagon-avoiding permutations. J. Algebraic Combin. 13(2), 111-136 (2001)

2. Billey, S.C., Jones, B.C.: Embedded factor patterns for Deodhar elements in Kazhdan-Lusztig theory. Ann. Comb. 11(3/4), 285-333 (2007)

3. Deodhar, V.V.: A combinatorial setting for questions in Kazhdan-Lusztig theory. Geom. Dedicata 36(1), 95-119 (1990)

4. Kazhdan, D., Lusztig, G.: Representations of Coxeter groups and Hecke algebras. Invent. Math. 53(2), 165-184 (1979)

5. Björner, A., Brenti, F.: Combinatorics of Coxeter Groups. Graduate Texts in Mathematics, vol. 231. Springer, New York (2005)

6. McLarnan, T.J., Warrington, G.S.: Counterexamples to the 0-1 conjecture, Represent. Theory 7, 181195 (2003) (electronic) 
7. Lusztig, G.: Cells in affine Weyl groups. In: Algebraic groups and related topics, Kyoto/Nagoya, 1983. Adv. Stud. Pure Math., vol. 6, pp. 255-287. North-Holland, Amsterdam (1985)

8. Lascoux, A.: Polynômes de Kazhdan-Lusztig pour les variétés de Schubert vexillaires. (French) [Kazhdan-Lusztig polynomials for vexillary Schubert varieties]. C.R. Acad. Sci. Paris Sér. I Math. 321(6), 667-670 (1995)

9. Lascoux, A., Schützenberger, M.-P.: Polynômes de Kazhdan et Lusztig pour les grassmanniennes. In: Young tableaux and Schur functors in algebra and geometry, Toruń, 1980. Astérisque, Soc. Math., vol. 87, pp. 249-266. France, Paris (1981)

10. Kazhdan, D., Lusztig, G.: Schubert varieties and Poincaré duality. Proc. Symp. Pure. Math., A.M.S. 36, 185-203 (1980)

11. Lakshmibai, V., Sandhya, B.: Criterion for smoothness of Schubert varieties in $\mathrm{Sl}(n) / B$. Proc. Indian Acad. Sci. Math. Sci. 100(1), 45-52 (1990)

12. Ryan, K.M.: On Schubert varieties in the flag manifold of $\mathrm{Sl}(n, \mathbf{C})$. Math. Ann. 276(2), 205-224 (1987)

13. Wolper, J.S.: A combinatorial approach to the singularities of Schubert varieties. Adv. Math. 76(2), 184-193 (1989)

14. Graham, J.J.: Modular representations of Hecke algebras and related algebras, Ph.D. thesis, University of Sydney (1995)

15. Marietti, M.: Boolean elements in Kazhdan-Lusztig theory. J. Algebra 295(1), 1-26 (2006)

16. Xi, N.: The leading coefficient of certain Kazhdan-Lusztig polynomials of the permutation group $S_{n}$. J. Algebra 285(1), 136-145 (2005)

17. Fokko du Cloux: http://igd.univ-lyon1.fr/ ducloux/coxeter/coxeter1/coxeter_e.html

18. Humphreys, J.E.: Reflection groups and Coxeter groups. Cambridge Studies in Advanced Mathematics, vol. 29. Cambridge University Press, Cambridge (1990)

19. Stembridge, J.R.: On the fully commutative elements of Coxeter groups. J. Algebraic Combin. 5(4), 353-385 (1996)

20. Cartier, P., Foata, D.: Problèmes Combinatoires de Commutation et Réarrangements. Lecture Notes in Mathematics, vol. 85. Springer, Berlin (1969)

21. Viennot, G.X.: Heaps of pieces. I. Basic definitions and combinatorial lemmas. In: Graph theory and its applications: East and West, Jinan, 1986. Ann. New York Acad. Sci., vol. 576, pp. 542-570. New York Acad. Sci., New York (1989)

22. Stembridge, J.R.: The enumeration of fully commutative elements of Coxeter groups. J. Algebraic Combin. 7(3), 291-320 (1998)

23. Green, R.M.: On rank functions for heaps. J. Combin. Theory Ser. A 102(2), 411-424 (2003)

24. Green, R.M.: Acyclic heaps of pieces. I. J. Algebraic Combin. 19(2), 173-196 (2004)

25. Green, R.M.: Acyclic heaps of pieces. II. Glasg. Math. J. 46(3), 459-476 (2004) 